\title{
Entretien avec Michèle Causse
}

\author{
Propos recueillis par Nadine Ltaif
}

In this 1999 interview recorded in "Trois Guinées ", Michèle Causse's and her lover's property in Lot, France, Causse gives us a sense of the trajectory of her literary career - from the friendship with Violette Leduc to her first work at the French literary magazine Les Temps modernes and L'Arc to her travels across languages: Italian and English (with which she is wellaquainted given her translation of writing by Djuna Barnes and Jane Bowles). "My writing is not hermetic," she insists. Its "difficulty" emerges from the fact that it translates a process of questioning, experimentation, patience and illumination.

N.L. Dans Violette Leduc, la biographie de Carlo Jansiti, vous évoquez le souvenir de Violette, que vous avez côtoyée, vous mentionnez qu'en votre compagnie, avec vous et Sabine, elle retrouvait son identité lesbienne. Pourquoi le mot retrouver? D'autre part, dans une correspondance vous m'avez aussi écrit : « il ne suffit pas d'être lesbienne pour faire un texte lesbien. »

M.C. C'est une très bonne question : Violette a été une pionnière en matière de sincérité intrépide, elle disait ce qui ne se dit pas. Et elle en a été bien punie. Je l'ai connue parce qu'elle a bien voulu m'apporter le manuscrit de Thérèse et Isabelle que je lui avais demandé de lire. Comme ce manuscrit était interdit de publication, à l'époque, j'avais écrit à Violette pour lui faire part de mon indignation.

Donc si tu veux, déjà par anticipation, je me sentais des affinités avec une femme dont les textes étaient interdits parce qu'ils énonçaient des vérités inaudibles. Violette était, pour moi, la personnification de la phrase de Virginia Woolf : «Lorsque les femmes parleront en leur nom, les hommes leur couperont la langue. »Cela n'a pas changé beaucoup, bien moins qu'on ne le croit. Ce blocage amène certaines auteures à l'autocensure, à une stérilisation, à un silence complet, parce qu'elles savent que de toute façon, il n'y a pas d'édition, de recension pour des femmes qui osent penser leur sexe. Pas leur sexe avec un petit $s$, leur Sexe avec un grand $S$, c'est-à-dire 
le genre qui est affecté à l'individu sexué au féminin : le genre minoré, le genre subsumé, le genre exploité, le genre approprié, et le genre opprimé. Ce fut longtemps le tabou absolu, parce que toute la civilisation, toute la société est fondée sur cette appropriation et cette exploitation, laquelle porte le nom de civilisation. Évidemment, il existe quelques empêcheuses de tourner en rond qui dénoncent. Mais à quel sort sont-elles vouées? Tu vas me dire « revenons à Violette, pourquoi dites vous qu'elle retrouvait sa fierté lesbienne? ». C'était un sentiment que j'avais fortement ancré en moi : lorsqu'elle était avec Sabine et moi, elle retrouvait une espèce d'identité non fabriquée, non sociale, non malheureuse, les échos de ses amours avec Hermine, avec Denise, ses amours les plus spontanées et joyeuses. Tant il est vrai qu'elle n'a pas supporté la rupture de notre couple. Elle l'a très mal vécue.

Pour le deuxième élément de ta question, désormais, nous vivons dans un monde où le lesbianisme est tout à fait in (je ne dirais pas à la mode). À tout le moins, une femme est bisexuelle, si elle veut connaître quelque chose de la vie. Ce qui ne passe vraiment pas, c'est le lesbianisme radical, à savoir une réflexion politique sur la contrainte à l'hétérosexualité, un lesbianisme assorti d'une conscientisation, ça ne passe pas, c'est censuré, ridiculisé. Au mieux on accepte les queers, ou tout ce que tu veux, queer et cuir, les dildos, les sex toys, les transsexuels, etc. On feint de lever un refoulé, un tabou. Bon, bon... Mais la pensée de l'oppression, de la dichotomie profonde qui régit le symbolique et le réel, cela ne doit point se dire. Ni a fortiori se combattre comme je le fais dans le langage. J'ai toujours dit que l'organe le plus interdit, le plus censuré, était le cerveau. Et pour cause.

\section{N.L. Violette Leduc vous a-t-elle marquée du point de vue littéraire?}

M.C. Non, pas vraiment. Même à vingt ans je l'admirais sans me sentir vraiment proche. Toutefois son intrépidité m'a été droit au coeur. L'Affamée est pour moi un des plus beaux livres d'amour qui existe. La Bâtarde ne m'a pas vraiment intéressée parce que je l'avais entendue de la bouche de l'auteure, si l'on peut dire, pendant des années. Le livre ne m'a pas requise littérairement. Mais L'Affamée, L'Asphyxie, Ravages, ces ouvrages haletants avaient provoqué en moi une sidération. Ils étaient uniques en leur temps.

N.L. Vous pensez que faire l'amour avec un homme exclut l'identité lesbienne? 
M.C. J'adore ta question. Elle te ressemble et tu ne dois surtout pas la changer. Écoute, moi j'ai pris position il y a longtemps, dans Trivia et d'autres revues. J'avais fait un parallèle avec l'assassinat. Que serait un assassin qui n'aurait pas assassiné ? Ou une lesbienne qui n'aurait pas couché avec une seule femme? On me rétorque en riant que c'est vraiment une définition a minima de l'identité lesbienne. Et je conviens que c'est une définition a minima. Le mot lesbienne, par définition, est relationnel, il implique qu'on soit au moins deux. Une lesbienne qui passerait sa vie dans l'abstinence, serait une lesbienne virtuelle, donc potentielle, ce qui me permet d'en venir au sujet qui m'est favori : qu'est-ce que le corps? Le corps c'est la clé d'or, c'est la clé de la connaissance. C'est pour cela qu'il est très important de savoir avec qui on échange du corps, autrement dit du sens et des sens. Si le corps n'est pas un vulgaire sac de peau mais un instrument de connaissance, le plus bel outil épistémologique, alors on ne doit pas le traiter comme quantité négligeable. On a soutenu qu'on pouvait s'allier à un homme ou une femme. Évidemment, on peut tout soutenir, tout et son contraire. Mais comment peut-on aimer son ennemi ? J'entends ennemi politique évidemment.

Nathalie Barney disait : « Women looks were my only books .» La connaissance se fait d'abord par la vie affective, la vie sensuelle. Toutefois, si tu restes exclusivement dans le domaine affectif tu ne vas pas très loin, dans cette société machiste, compétitive et capitaliste; donc il est capital que l'amour lesbien soit gynophile et travaille à l'advenir d'une socialité totalement différente de celle qui régit à l'heure actuelle les corps sexués.

\section{N.L. Dites deux autres affinités littéraires?}

M.C. Les Américaines m'ont beaucoup marquée. Djuna Barnes, évidemment. Quand j'ai une affinité littéraire avec une auteure, je lui écris. J'ai une vraie correspondance. J'ai eu beaucoup d'admiration pour Anne Hébert, que je suis allée voir quand j'avais vingt ans, avec beaucoup d'audace et de mauvais poèmes. Elle s'est comportée comme il convient quand on a quarante ans et qu'on voit débarquer une jeune fille un peu exaltée qui vient vous faire une déclaration d'amour détournée. Djuna Barnes ou Anne Hébert, si tu veux, étaient pour moi de beaux réceptacles physiologiques du verbe. Et donc j'en étais amoureuse. Bien que je ne sois pas juive, le Livre est Tout pour moi. Sur le plan littéraire, tous les styles forts m'intéressent. Il peut s'agir aussi bien de Gertrude Stein que de Beckett. De Dante ou de Melville. Mais je peux dire que, désormais et depuis vingt ans, ce sont les 
idées, les concepts qui me donnent de vrais orgasmes de lecture. Lorsqu'une idée est nouvelle, lorsqu'un concept, que ce soit en sociologie, en philosophie, en anthropologie me parait pertinent, alors là j'ai des vrais élans de vitalité, des vrais bonheur de lecture. C'est rare, trop rare.

N.L. Comment viviez-vous la société littéraire française des années soixante, autour d'André Breton, Sartre et Simone de Beauvoir? Et quels étaient les lieux lesbiens de l'époque?

M.C. Je précise que de 1963 à 1972, je vivais en Italie. Donc la société littéraire française, je ne la connaissais pas, si ce n'est dans le texte. Toutefois, à vingt ans, alors que je vivais encore à Paris, j'ai été contactée par Bernard Pingaud - du comité de rédaction des Temps modernes - pour travailler avec lui à la revue L'Arc. Je connaissais un peu Simone de Beauvoir, puisque c'était elle qui avait incité Violette Leduc à me répondre, (tant il est vrai que sans Simone de Beauvoir, Violette Leduc n'aurait jamais répondu à mes lettres), j'étais donc marginalement dans la mouvance des Temps modernes. Je travaillais très modestement pour $l^{\prime}$ Arc : j'écrivais des lettres, je tapais à la machine, j'étais dans la subalternité juvénile. Cela m'a beaucoup appris. J'ai vu de près que les hommes les plus doués de leur génération étaient, sur le plan affectif, humain, de grands singes, des primates. Et je me suis dit « comment peut-on vouloir des relations avec des primates inférieurs ? " Je les trouvais d'une grossièreté, d'un simplisme, d'un autisme! Sur le plan informationnel, les hommes sont toujours imbattables, mais sur le plan relationnel, en France ou en Italie, ce sont de grands phallus ambulants, sujets aux bimbo eruptus en raison desquels les femmes ne sont pour eux que de la fermme.

Le monde littéraire de l'époque était castrateur dans la mesure où régnait l'école du regard. Je ne sais pas si on se souvient au Québec de ladite école, publiée aux Éditions de Minuit et dont le représentants les plus célèbres sont Robbe-Grillet et le prix Nobel Claude Simon, mais les ouvrages de ces auteurs étaient pour moi la quintessence de l'ennui, du bannissement de toute subjectivité. Des êtres qui voulaient écrire au « je » ont beaucoup souffert de la tyrannie littéraire du moment. Il n'y avait pas de mouvement féministe, il ne faut surtout pas l'oublier. Je me souviens avoir été horrifiée par une phrase de Beauvoir qui disait, en 1958, que le féminisme était dépassé ! C'est une phrase qui m'avait causé un véritable électrochoc. Moi, j'ai commencé à croire dans mon écriture avec le mouvement de libération des femmes. Tu sais, on a dit que, sans la 
Révolution française, Robespierre serait resté un obscur avocat, eh bien, sans le MLF tel que je l'ai vécu en Italie, pas de Michèle Causse. J'aurais laissé mes textes dans un tiroir. Je n'aurais pas eu de rapport d'adresse. Je n'aurais peut-être même pas pu écrire.

N.L. Ma question à présent concerne vos voyages, et votre métier de traductrice : Italie, Tunisie, États-Unis, Martinique, Québec. Quel a été l'apport de ces voyages à votre vie d'écrivaine?

M.C. Il y a eu deux types de voyages. Les voyages que j'ai fait par amour, ceux où j'ai suivi, voire poursuivi, un être que j'aimais. C'est le voyage fortuit ou concerté, lié à un être humain qui se trouve à tel endroit géographique, disons Rome ou New York. Et puis il y a eu les voyages de désespoirs successifs ou des voyages de travail qui sont liés à des lieux de fuite, des lieux où je vais pour ne pas sombrer, pour ne pas mourir. Le Québec en fait partie, puisque sans le Québec je n'aurais pas survécu matériellement ni symboliquement. Partant, mon rapport avec les pays est évidemment très différent. Ce n'est plus l'excitation, la légèreté, c'est la souffrance : de toute façon, quand on est une immigrante, quand on a été privée de tout, de domicile, de sécurité, on entre dans un savoir que n'ont pas les êtres qui n'ont pas immigré. Moi je ne divise pas les populations en Nord-Sud, etc., je les divise entre celles qui ont immigré et celles qui n'ont jamais eu à le faire.

Le voyage qu'on fait par choix n'a rien à voir avec les voyages qu'on fait par nécessité, même si les deux concourent au même destin, sont en quelque sorte un fatum. Le rapport de mon corps, de ma tête, à ces pays-là n'est pas le même là où te précède ton coeur. Je ne serais pas celle que je suis sans les voyages, c'est certain. Être à l'étranger vous change, vous force à vous assouplir, car les codes ne sont pas les mêmes, exigent une forme d'adaptation à chaque fois différente. Lorsqu'on est immigrante, de surcroît, on est traitée comme telle par les habitants d'un pays. On perd beaucoup d'amies, des êtres qui vous aimaient quand vous étiez touriste. On n'est plus perçue du tout de la même manière, on se sent infériorisée. En France maintenant, quand je vois des immigrés, je n'ai pas le même regard qu'avant. J'ai beaucoup plus de considération, de compassion et d'attention, de vraie solidarité. Je considère l'émigration comme une étape initiatique, un revers qui apporte beaucoup pour la compréhension de soi et des autres. J'avais d'ailleurs dit à Michèle J. - lorsqu'elle était à Ottawa - qu'il fallait créer un organisme qui prenne en charge l'écoute des 
immigrés arrivant de pays où ils avaient vécu des tragédies collectives ou privées. Un pays comme le Québec, qui n'a pas connu de guerre, un pays de bonheur tranquille, ne peut pas avoir de véritable écoute pour les tragédies, à la fois historiques, externes aux individus, et pour la complexité des intériorité malmenées. On est dans un grand silence au Québec. Même quand on vous donne une place d'émigrée. Quelle est la Kurde, la violée algérienne, qui va être entendue, aussi pleins de bonne volonté que soit la Québécoise ou le Québécois qui vont l'accueillir? Ce n'est la faute de personne, manque l'expérience, l'imagination du malheur. Les gens qui sortent de camps de concentration se sont toujours plaints de ne pas rencontrer d'oreille. Ils ne se plaignaient pas, d'ailleurs, ils disaient : " on ne peut pas en parler, personne ne peut entendre .» Et c'était vrai. Au Québec, je trouve qu'on devrait mettre des émigrés à l'accueil des émigrés.

N.L. Ça commence je crois en ce qui a trait à la prise en charge du langage. Il y a un côté hermétique à votre écriture dont seuls les initiés parviennent à saisir le sens. Pourquoi avoir choisi de développer un style si hermétique et savant ? Pour qui écrivez-vous ? N'est-ce pas là une façon bien à vous de vous masquer derrière un jeu de langage qui érige une forteresse difficile d'accès, pour décourager ceux qui tentent d'y forcer le sens, ou pour rendre impuissante toute tentative phallique de récupération?

M.C. Une façon de ne pas décourager la lecture, de faire comprendre le sens, comme tu dis, c'est de recourir à l'essai. J'ai écrit des textes que je trouve simples: L'Interloquée; les oubliées de l'oubli; délgénérées, Le monde comme volonté et représentation. Dans ces essais, voire même le dernier Contre le sexage, j'ai tenté d'expliciter les concepts qui me tenaient à coeur et que j'illustrais dans mes textes de fiction. Maintenant, qu'on me reproche de ne pas écrire comme je parle dans mes romans (voir ( ) ou Voyages de la Grandes Naine en Androssie) relève pour moi d'un malentendu classique, fort bien compris par Gertrude Stein qui disait : « Writing means more for the writer than for the reader. » C'est une question de déontologie, de fidélité à soi, et plus encore à la langue, un risque pris et assumé face à l'incompréhension probable du lectorat, face au rapport qui va se révéler manquant, défaillant. Il appartient à la lectrice de résister ou de s'imposer l'effort d'accéder à l'histoire que je vis avec les mots, dans mon corps à corps avec le langage. La lectrice est libre d'ignorer mon aventure humaine et langagière. Elle est libre de la trouver élitiste mais pour moi, 
qui la vis de l'intérieur, elle s'impose d'elle-même. Lorsque je parle, je suis dans la spontanéité de l'oral, dans l'approximatif, dans la joie de la présence du corps de l'autre, de toi en ce moment; dans l'écriture, je suis dans l'élucidation perplexe, dans la spéléologie. Les Italiens avaient intitulé une revue littéraire Marcatré. Le marcatré, c'est la profondeur à laquelle on trouve le pétrole, et pour moi l'écriture c'est le moment où l'on va aussi loin que possible dans l'élucidation et dans la recherche et la découverte d'une écriture, d'un style. Lequel peut et doit varier d'une oeuvre à l'autre. L'auteure creuse son puits. Et à une certaine profondeur, elle trouve son pétrole. Et moi mon pétrole, c'est ce que tu dis être un matériau décourageant ou dissuasif, etc. Hélas, il faudrait me couper la tête pour me changer. On peut dire que je suis élitiste, on a le droit de le dire, cela ne me concerne pas. Est-ce qu'on va dire en parlant de la cathédrale saugrenue de Gaudi : " Ah c'est élitiste, une cabane de berger au fond d'un pré, c'est vachement mieux. "Non, on trouve que c'est un travail intéressant, bizarre peut-être, hermétique aussi, mais enfin, dans les arts plastiques, on ne parle pas d'élitisme quand on ne comprend pas ce qu'on voit. Pourquoi le mot ne serait-il pas ce qu'est le pot de peinture pour l'artiste ? Ici, n'oublions pas qu'il s'agit de renouveler le langage, car il existe une vraie nécessité pour un être sexué au féminin de réinventer complètement sa langue, et de plier à sa propre glorification un langage qui la veut anéantie, silencieuse, néantisée, objectifiée, réifiée. Je travaille avec des pioches des pelles et des pics. Je trouve légère l'accusation d'élitisme. On la prête souvent à des langages difficiles, parce que cette « difficulté » est la traduction d'un operare, comme disent les Italiens, d'un oeuvrer à quelque chose, d'un travail sur quelque chose, qui implique beaucoup de questionnements, de tâtonnements, de patience et de fulgurances. Mais on a du mal à en parler, parce que ça fait tellement écrivain maudit. Tout texte qui interroge, remet en cause, est le produit d'un être profondément endolori par le monde dans lequel il vit et qu'il veut changer. René Char disait que quiconque ne vient pas au monde pour le changer ne mérite pas de vivre. Toutefois même quand mon énoncé est désespéré - et il l'est toujours - je m'applique à être jubilatoire dans l'énonciation. J'ai un peu de mal à l'heure qu'il est car j'écris dans un moment où la guerre dans les Balkans a commencé. Dans un moment où j’ai du mal à trouver de la joie, si ce n'est dans les épiphanies de la nature, le soleil, les fleurs, les petits enfants. Mais si je prends la société at large, je suis un peu désespérée que la voix des féministes ne soit pas suffisament entendue. Je fais des 
distinguo entre les femmes, les féministes et les lesbiennes. Je vois des étapes de conscientisation. Les femmes sont le troupeau sur lequel campent les hommes, le troupeau généreux, la matière première sur laquelle le reste de l'humanité campe, les femmes qui nettoient derrière les hommes qui tuent, puis les féministes qui ont pris conscience qu'il existe des classes de sexe, et enfin les lesbiennes radicales, les hérétiques de la pensée hégémonique, celles qui ont fui le phallogocentrisme, qui disent « le roi est nu », celles qui font disparaître l'apartheid des genres. À mon sens, nous sommes dans la protohistoire de l'espèce humaine. Toutefois je crains qu'on passe directement au stade du clonage subhumain. Comme tu disais hier, « ça n'a pas d'importance que je sois Libanaise, Québécoise, etc., je suis Nadine. » Eh bien c'est ce à quoi je voudrais arriver, à l'individu qui, sur terre, aurait les mêmes droits et devoirs indépendamment de sa sexuation, de sa couleur, de sa fortune. Le droit de respirer, le droit de vivre, le droit d'avoir un toit sur la tête, le droit d'avoir des droits. Je ressens fortement le sort des femmes du Tiers-monde. Crois-tu qu'on puisse vivre heureuse sachant que les femmes des Talibans ne peuvent sortir, travailler pour se nourrir, qu'on ne les soigne pas, qu'on ne les instruit pas et qu'elles se suicident? Qui s'en soucie? Les hommes, et pour cause, ne s'élèvent pas en masse contre le stillicide des femmes, contre leur réduction en choses muettes et aveugles. C'est dans la logique extrême de leur système. Avant la guerre du Kosovo, je m'étais dit, " tiens, on va d'abord faire partir les femmes et les enfants, puis ensuite on va laisser les hommes jouer à la mort avec leurs fusils, leurs avions, leur poison, enlevons les femmes et les enfants des pays où sévissent les hommes, et mettons-les à l'abri ". Il n'y a rien de plus triste que de voir ces veuves, ces femme de la Place de Mai, en Argentine, circuler en rond sur cette place avec les photos de leurs hommes. Leurs hommes, ils ont été détruits par d'autres hommes : ce ne sont pas des femmes qui les ont détruits. Il est grand temps qu'on fasse advenir un autre discours que celui de la mort, de la valorisation mercantile de la destruction. Il est peut-être même déjà trop tard. Mais avec l'alpha, dans Contre le sexage, Éliane Pons et moi faisons une proposition linguistique à la fois politique et pacifiste pour que coexistent sur la terre des sujets à égalité, qui soient une fin et non un moyen. 


\section{Ouvrages cités}

Jansiti, Carlos. Violette Leduc. Paris : Grasset et Fasquelle, 1999.

\section{Euvres de Michèle Causse}

L'Encontre. 1975. Paris : Éditions des femmes. (roman)

Écrits, voix d'Italie. 1977. Paris : Éditions des femmes. (anthologie)

L'Intruse. 1980. Paris : Éd. Le Nouveau Commerce. (poésie)

Berthe ou un demi-siècle auprès de l'Amazone. 1980. Paris : Éd. Tierce. (biographie)

Stèles de Jane Bowles. 1982. Paris : Éd. Le Nouveau Commerce.

Lesbiana, Seven Portraits. 1980. Éd. Le Nouveau Commerce. (nouvelles)

Lettres à Omphale. 1984. Paris : Éd. Denoël-Gonthier. (correspondance)

( ). 1987. Montréal : Éd. Trois. (roman)

À quelle heure la levée dans le désert? 1989. Montréal : Éd. Trois. (théâtre)

«L'Ilote ». 1990. Montréal : dans Trois. (nouvelle)

Voyage de la Grande Naine en Androssie. 1993. Montréal : Éd. Trois. (roman)

«Duelle ». 1994. Montréal : dans Treize. (nouvelle)

«Court of Appeal ». 1996. Montréal : dans Tessera 21, « La violence symbolique et l'avant-garde ».

Quelle lesbienne êtes-vous? 1997. Paris : Éd. Paroles de lesbiennes.

\section{Essais}

Contre le sexage. 2000. Paris : Balland.

Petites réflexion sur Bartleby. 1980. Paris : Éd. Le Nouveau Commerce.

« Le monde comme volonté et représentation ». 1986. Montréal : Éd. St-Martin.

(Traduction en anglais : "The world as will and representation" in Jeffner Allen, Philosophy and Cultures, SUNY P).

L'Interloquée; les oubliées de l'oubli; dé/générée. 1990. Montréal : Éd. Trois.

«Dire du corps, corps du dire». 1979. Journal d'une femmes soumise. Paris : Flammarion.

« Rencontre avec Djuna Barnes ». 1982. L'Almanach des dames. Paris : Flammarion.

\section{Enregistrement sonore sur cassette}

A la lettre. 1989. Montréal : prod. Anne-Marie Alonzo, extrait d'oeuvres lus par l'auteure.

Film

Corps de parole. 1989. Montréal. (de Suzanne Vertue et Diane Effernan). 